Title:

Modeling and Simulation for the

Semiconductor and Optoelectronic Industries

Author(s):

Submitted to:
David Cartwright, T

Robert Albers, T-11

Denise George, $\mathrm{T}-1$

Mark Gray, CIC-19

Joel Kress, T-12

Thomas Kwan, XPA

Richard Nebel, T-15

Patrick Soran, XTM

Galen Straub, T-1

Harold Trease, XHM

Steven Valone, MST-7

DOE Office of Scientific and Technical

Information (OSTI)

\section{Los Alamos}

NATIONAL LABORATORY

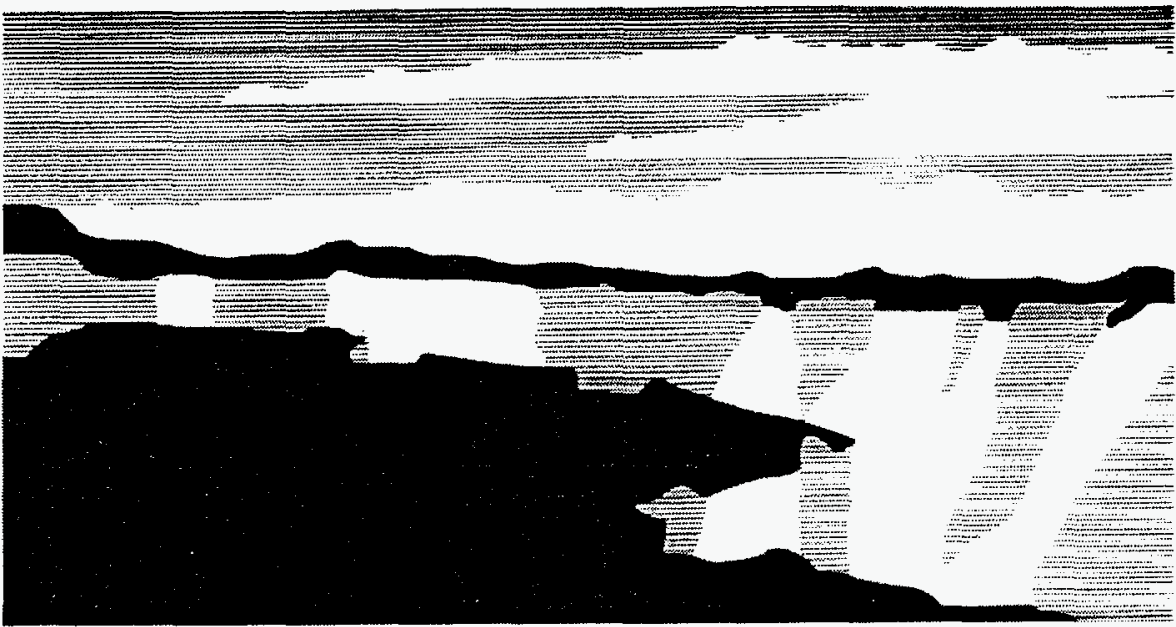

Los Alamos National Laboratory, an affirmative actionequal opportunity employer, is operated by the University of Catifornia for the U.S. Department of Energy under contract W-7405-ENG-36. By acceptance of this article, the publisher recognizes that the U.S. Government retains a nonexclusive, royaltyfree license to publish or reproduce the publshed form of this contribution, or to allow others to do so, for U.S. Govemment pumposes. The Los Alamos National Laboratory requests that the publisher identity this article as work performed under the auspices of the U.S. Department of Energy. 


\section{DISCLAIMER}

Portions of this document may be illegible in electronic image products. Images are produced from the best available original document. 


\title{
Modeling and Simulation for the Semiconductor and Optoelectronic Industries
}

\author{
David Cartwright*, Robert Albers, Denise George, Mark Gray, Joel Kress, Thomas Kwan, \\ Richard Nebel, Patrick Soran, Galen Straub, Harold Trease, and Steven Valone
}

\begin{abstract}
This is the final report of a two-year, Laboratory-Directed Research and Development (LDRD) project at the Los Alamos National Laboratory (LANL). Semiconductor technology development costs and time-to-market pressures are rapidly increasing with each new technology generation. However, computeraided integrated circuit (IC) design tools have been shown to reduce IC design time and overall costs, and the micro- and opto-electronics industries have come to believe that enlarging the modeling scope to include new materials processing simulators will have a corresponding profound impact on manufacturing. This project sought to assess the feasibility of building a suite of predictive advanced technology modeling and simulation tools for use by the semiconductor industry.
\end{abstract}

\section{Background and Research Objectives}

Integrated circuit (IC) manufacturing technology has developed faster than the capability to model and simulate its various components. On the other hand, IC computer aided design (CAD) tools, developed largely by Semiconductor Research Corporation (SRC) universities within the past decade, which model the product and simulate its performance, have been successfully applied to minimize IC design time and cost. Although CAD applied to IC design has been extremely successful, dramatically demonstrating the power of the approach, there still remains much to be accomplished, not only for IC CAD, but more importantly for process modeling. The cost and complexity of IC manufacturing has now reached the level where acquisition and application of advanced modeling and simulation tools is an imperative. The Semiconductor Industry Association (SIA) National Semiconductor Technology Road Map identifies technology modeling and simulation as an increasingly important tool in every Road Map area.

The objective of this project was to assess the feasibility of building a suite of predictive advanced technology modeling and simulation tools for use by the semiconductor industry.

\footnotetext{
* Principal investigator, e-mail: dcc@lanl.gov
} 
These tools would alleviate much of the need for costly and time consuming industrial laboratory experiments to develop each new technology generation empirically. The ultimate goal is an improved ability to model and simulate semiconductor materials, devices, systems and manufacturing processes. The five year goal for the industry is to achieve a complete suite of predictive simulators for the 0.1 micron technology generation, which the SIA Road Map forecasts to be introduced into manufacturing in 2007. To meet this goal successfully, advanced modeling and simulation tools must be in the hands of the industrial engineers before the end of this century, with initial results beginning during 1994 and culminating in a specified suite of modeling and simulation tools by 1999. The achievement of the fifth year program goal will be measured by the capability of the simulation tools to model the 0.1 micron products and their manufacturing technology in a predictive manner, as judged by the semiconductor industry.

\section{Importance to LANL's Science and Technology Base and National R\&D Needs}

This project supports Los Alamos core competencies in theory, modeling, and highperformance computing as well as complex experimentation and measurement. It enhances the Laboratory's visibility and ability to respond to initiatives in modeling and simulation on semiconductors.

\section{Scientific Approach and Results to Date}

The technical thrust areas to be evaluated were (1) topography models, (2) bulk processing, and (3) grids and computational science.

\subsection{Topography Models}

Topography simulators, combined with sufficiently detailed chemistry derived from either computational or experimental methods, can be used to provide insight into how surfaces are affected during deposition and etch processes. The topography thrust area therefore focused on the development of capability for fully three-dimensional modeling of feature evolution by either deposition or etch processing.

Reactive Processing of Microelectronics: Initial calculations have been completed on benchmarking density functional approaches for computing thermochemical properties of $\mathrm{Si}-\mathrm{H}$ $\mathrm{Cl}$ molecules. The conclusion of this initial study is that gradient-corrected functionals are capable of giving more accurate thermochemical information than traditional quantum chemical approaches. However, an analogous procedure to the BAC-MP4 method will still be needed to 
correct the remaining errors to obtain thermochemistries and to estimate rate constants. Another series of calculations has been initiated involving $\mathrm{Si}-\mathrm{Cl}$ species to aid the development of interatomic potentials for simulations of etching processes. Quantum chemical calculations on $\mathrm{Si}_{\mathrm{n}} \mathrm{Cl}_{\mathrm{m}}$ gas phase species and eventually on $\mathrm{Cl}$-containing $\mathrm{Si}$ clusters have been carried out to obtain bond energies as a function of the number and types of neighboring atoms around each $\mathrm{Si}$ that can be used in deriving interatomic potentials. Tersoff and Stillinger-Weber potentials have been fitted for hydrogen. Hydrogen is being used as a test case in determining some of the hazards of the fitting process in regards to surface kinetics. Hydrogen has the greatest amount of and most accurate data of any system under consideration for the project.

Electronic structure: High accuracy (QCISD) calculations on $\mathrm{Cl}_{2}$ and $\mathrm{Cl}_{3}$ have been done to benchmark gradient-corrected density functional theory (DFT) techniques. The latter will feed into new $\mathrm{Cl}-\mathrm{Cl}$ interatomic potentials under development. Calculations on $\mathrm{Si}_{\mathbf{x}} \mathrm{H}_{\mathbf{y}} \mathrm{Cl}_{\mathbf{Z}}$ molecules have been carried out using all-electron (up to $\mathrm{x}=3$ and $\mathrm{z}=3$ ) and valence-electron (with ECPs, up to $\mathrm{x}=5$ and $\mathrm{z}=3$ ) techniques to assess any errors associated with ECPs in DFT calculations in calculating gas phase and surface thermochemistries. Scaling tests on $\mathrm{SigH}_{14}$ and $\mathrm{Si}_{21} \mathrm{H}_{26}$ cluster models have been performed on IBM and HP workstation clusters; such architectures will be important for calculations on large molecules. Calculations on $\mathrm{H}$ and $\mathrm{Cl}$ binding to clusters from $\mathrm{SigH}_{14}$ through $\mathrm{Si}_{49} \mathrm{H}_{44}$, which represent the $\mathrm{Si}(100)$ surface, have been carried out using semi-empirical techniques. The goal was to observe how the calculated thermochemistries vary with the total number of atoms in the cluster and with the number of atoms in the cluster that are allowed to relax from their lattice positions.

\subsection{Bulk Processing}

The industry presently has process simulators, based on continuum PDE approaches, that can predict dopant and defect behavior based on a set of assumptions concerning the atomic mechanisms involved. What is not known is the exact form these assumptions should take and how to determine parameters in the models based on these assumptions.

Measurements are very difficult because there is no way to measure the mechanisms directly and because many different mechanisms can be used to explain a given experiment.

The ultimate goal of this portion of the project was to supply a physically-based, predictive code capable of calculating ion damage and amorphization as a function of dosage and ion energy, defect creation and annihilation, transient enhanced diffusion, and effects on dopant and defect concentrations due to thermal annealing. Dopant concentrations need to be accurately determined in both two and three dimensions at each step in the process, including both implant range and lateral straggle. Multiple targets (implanting through other layers) and multiple implants (dopant-dopant interactions) also needed to be addressed.

Dopant \& Defect Diffusion: 
- We completed all-electron, full-potential total-energy calculations of the vacancy formation energy in 64-atom supercells of Si. The results for the vacancy energy are in agreement with recent calculations: $3.7 \pm \sim 0.1 \mathrm{eV}$. The first-neighbor relaxation is $-10 \%$; the radial component of second-neighbor relaxation is $-1.3 \%$. Calculations of the vacancy formation energy in 128-atom Si cells are continuing.

- Calculations of lattice relaxation and relaxation energy around As and $P$ impurities are completed. The first-neighbor relaxation is approximately $+3 \%$ in As and $-0.2 \%$ in $P$.

- Calculations of the energy of a Si interstitial along the tetrahedral-hexagonal path have been continued.

- Work has been continued on the grain boundary structure of Si. One grain boundary seems to be over bonded ( 5 -fold instead of the usual 4-fold bonded tetrahedra), which would imply that the grain boundary might be intrinsically electrical inactive. The electrical activity might come from the segregation of vacancies, interstitials, or impurities.

Ion Implantation: On June 3, 1995, version 2.0 of the UT-Marlowe ion implantation code was released to more than 150 users and interested individuals at industrial research organizations, universities, and national laboratories. This was very close to the original target release date of May 1995, and in fact, the version 2.0 incorporated a number of features which were not initially planned for this release, but were finished ahead of schedule. Los Alamos played a substantial role in improving the UT-Marlowe code and the significant innovations in Version 2.0 of UT-Marlowe can be summarized as follows:

- Interatomic pair potentials and a local electronic stopping model for arsenic have been incorporated, adding arsenic as another implant species.

- A damage model for arsenic has been implemented and general improvements have been made to the damage physics calculation. Detailed comparisons with SIMS experimental data have been used to fine-tune the model, assuring accurate predictions over a broad parameter range.

- A simple noise reduction scheme has been developed, which will serve as the starting point for future variance control and optimization.

- A suite of test problems was developed to exercise all major physics options, and these problems were used to validate the code on all platforms except the Alpha. We need to obtain access to an Alpha in order to perform the necessary validation tests.

- Portable Pre-Processor for Fortran Codes: Because the standard UNIX CPP pre-processor is not satisfactory for use with Fortran codes, we have now developed a small, fully portable pre-processor that works identically on all systems and is adapted for use with Fortran codes. The directives are similar to, but more general than, CPP, providing additional capabilities for Boolean selection of conditional code and several other useful 
features. This pre-processor may be used with future releases of UT-Marlowe and may have other potential applications as well.

- Portable Random Number Generator for UT-Marlowe: We incorporated a portable random number generator into UT-Marlowe to allow comparison testing on different platforms with identical random number sequences. This generator was not included in the Version 2.0 release, but could easily be incorporated into future releases with the aid of the new preprocessor.

- MOCABULK Monte Carlo Device Modeling Code: The MOCABULK Monte Carlo electron-hole transport code from the University of Illinois was installed at Los Alamos. We have now linked MOCABULK to the Los Alamos three-dimensional graphics/analysis system using a standard data output library developed for CRADA applications. Several extensions and enhancements were added to the library. All of the diagnostics in MOCABULK can now be accessed and viewed with the Los Alamos graphics/analysis code.

\subsection{Grids and Computational Science}

Mesh generation is at the heart of any semiconductor simulation code. At present process and device simulations are primarily confined to two dimensions. The extension of these simulation codes to three dimensions will require the ability to automatically generate and optimize three-dimensional moving surface and volume grids as well as local adaptive mesh refinement to resolve physical phenomena. The national laboratories have extensive experience in this area, especially three-dimensional grid generation and modification, and should play a important role in the development of the next generation simulators.

The goal of the grid thrust-area is to develop utilities for mesh generation, adaptive mesh refinement, dynamic mesh reconnection, and moving adaptive mesh algorithms contained in a software package called X3D. To ensure widespread use, implementation of these algorithms should be done through a GRID TOOLBOX that is functionally capable of being called from various codes but physically separated from physics codes. The following results were obtained:

- New algorithms to implement sheet surfaces were developed.

- Structured programming standards were applied to critical parts of X3D. These changes were necessary to isolate the parts of X3D that should remain invisible to the user from those parts that may be modified by the user.

- Specification of the test suite to be used to verify code was continued.

- Three-dimensional (error gradient) smoothing algorithms and two-dimensional elliptical smoothing algorithms were improved. The two-dimensional algorithm uses a volume average and scaled reference field. 
Benchmarks and test problems: The Poisson solver is now up and running in X3D and running a case with an analytic solution for comparison of relative error. We are checking various refinement tests to minimize error and to gain insight on refinement based on physics tests and geometric tests. The refinement of the grid for more accurate solution is based on the solution from the first grid. We have completed some analytic work on the near boundary expansion of our test case Poisson problem.

\section{DISCLAIMER}

This report was prepared as an account of work sponsored by an agency of the United States Government. Neither the United States Government nor any agency thereof, nor any of their employees, makes any warranty, express or implied, or assumes any legal liability or responsibility for the accuracy, completeness, or usefulness of any information, apparatus, product, or process disclosed, or represents that its use would not infringe privately owned rights. Reference herein to any specific commercial product, process, or service by trade name, trademark, manufacturer, or otherwise does not necessarily constitute or imply its endorsement, recommendation, or favoring by the United States Government or any agency thereof. The views and opinions of authors expressed herein do not necessarily state or reflect those of the United States Government or any agency thereof. 